\title{
Interplay of spherical closed shells and $N / Z$ asymmetry in quasifission dynamics
}

\author{
G. Mohanto, ${ }^{1, *}$ D. J. Hinde,${ }^{1, \dagger}$ K. Banerjee,,${ }^{1,2}$ M. Dasgupta, ${ }^{1}$ D. Y. Jeung, ${ }^{1}$ C. Simenel, ${ }^{1}$ E. C. Simpson,,${ }^{1}$ A. Wakhle,${ }^{1,}$ \\ E. Williams, ${ }^{1}$ I. P. Carter,${ }^{1,}$ K. J. Cook, ${ }^{1}$ D. H. Luong, ${ }^{1, \|}$ C. S. Palshetkar,,${ }^{1, \mathbb{I}}$ and D. C. Rafferty ${ }^{1}$ \\ ${ }^{1}$ Department of Nuclear Physics, Research School of Physics and Engineering, Australian National University, \\ Australian Capital Territory 0200, Australia \\ ${ }^{2}$ Variable Energy Cyclotron Centre, 1/AF-Bidhannagar, Kolkata 700064, India
}

(Received 19 January 2018; revised manuscript received 12 April 2018; published 9 May 2018)

\begin{abstract}
Background: Quasifission (QF) has gained tremendous importance in heavy-ion nuclear physics research because of its strong influence on superheavy-element synthesis. Collisions involving closed-shell nuclei in the entrance channel are found to affect the QF reaction mechanism. Hence, it is important to improve the understanding of their effect on QF. Apart from that, some recent studies show that the difference in $N / Z$ of reaction partners influences the reaction dynamics. Since heavier doubly magic nuclei have different $N / Z$ than lighter doubly magic nuclei, it is important to understand the effect of $N / Z$ mismatch as well as the effect of shell closures.

Purpose: To investigate the effect of entrance-channel shell closures and $N / Z$ asymmetry on QF. The reactions were chosen to decouple these effects from the contributions of other entrance-channel parameters.

Method: Fission fragment mass-angle distributions were measured using the CUBE fission spectrometer, consisting of two large area position-sensitive multi-wire proportional counters (MWPCs), for five reactions, namely, ${ }^{50} \mathrm{Cr}+{ }^{208} \mathrm{~Pb},{ }^{52} \mathrm{Cr}+{ }^{206,208} \mathrm{~Pb},{ }^{54} \mathrm{Cr}+{ }^{204,208} \mathrm{~Pb}$.

Result: Two components were observed in the measured fragment mass angle distribution, a fast mass-asymmetric quasifission and a slow mass-symmetric component having a less significant mass-angle correlation. The ratio of these components was found to depend on spherical closed shells in the entrance channel nuclei and the magnitude of the $N / Z$ mismatch between the two reaction partners, as well as the beam energy.

Conclusions: Entrance-channel spherical closed shells can enhance compound nucleus formation provided the $N / Z$ asymmetry is small. Increase in the $N / Z$ asymmetry is expected to destroy the effect of entrance-channel spherical closed shells, through nucleon transfer reactions.
\end{abstract}

DOI: 10.1103/PhysRevC.97.054603

\section{INTRODUCTION}

Nuclei having certain numbers of neutrons and protons are more strongly bound than neighboring nuclei. These are referred to as closed-shell nuclei or sometimes as "magic nuclei" in the literature. The nuclear shell model can explain these magic numbers up to $Z=82, N=126$. But the prediction of the next spherical shell closure is model dependent. Different models predict the next proton shell closure to be between $Z=114$ and 126 [1-4] and neutron shell closure at

\footnotetext{
*Present address: Nuclear Physics Division, Bhabha Atomic Reserch Centre, Mumbai 400085, India.

†David.Hinde@anu.edu.au

${ }^{\ddagger}$ Present address: Cyclotron Institute, Texas A\&M University, College Station, Texas 77843, USA.

${ }^{\S}$ Present Address: CSIRO Sorting and Sensing, Mineral Resources, Building 67, ANSTO, Lucas Heights, Australia.

"Present address: Scandinavian Health Limited, Taiwan Branch, 136 Guosheng 2nd Street, Taoyuan District, Taoyuan City, Taiwan 330.

"Present Address: Tata Institute of Fundamental Research, Mumbai 400005, India.
}

$N=184$. An increased decay lifetime for nuclei in this region is predicted due to shell stabilization.

Intense efforts have been made in the past few decades to produce superheavy elements (SHE) near the next shell closures using heavy ion fusion reactions $[2,5,6]$. Experiments either use the spherical magic target nuclei ${ }^{208} \mathrm{~Pb}$ (doubly magic) and ${ }^{209} \mathrm{Bi}$ with suitable projectiles in cold-fusion reactions $[7,8]$ or the spherical doubly magic projectile ${ }^{48} \mathrm{Ca}$ on suitable actinide target nuclei $[9,10]$ in hot-fusion reactions. Many new elements have been discovered, the latest being $\mathrm{Nh}$ $(Z=113)$, Mc $(Z=115)$, Ts $(Z=117)$, and $O g(Z=118)$ $[11,12]$. However, the production cross sections for SHE are very small and hence it is crucial to understand the reaction mechanism so that the optimal combination of projectile and target nuclei can be used.

The main challenges in SHE production are the fission processes which suppress the evaporation residue (ER) cross sections. The two different modes of fission that reduce the ER formation probability are compound nucleus fission (CNF) and quasifission $(\mathrm{QF})$. In the case of $\mathrm{CNF}$, the compound nucleus $(\mathrm{CN})$ is formed but often does not survive due to repulsion between the huge number of protons and breaks in two fragments, often of comparable masses. 
Survival against compound nuclear fission depends on excitation energy, since fewer steps in fission-evaporation competition should greatly favor superheavy-element synthesis. QF, on the other hand, is a much faster process where targetlike and projectile-like nuclei separate soon after capture; thus, the system does not fuse to form a compact $\mathrm{CN}$.

The contribution of quasifission becomes more significant as the Coulomb repulsion quantified by the charge product $Z_{p} Z_{t}\left(Z_{p}\right.$ is projectile atomic number and $Z_{t}$ target atomic number) of the reaction partners increases. Hence, $Q F$ is more important for very heavy nucleus formation [13-15]. A number of studies showed that QF also depends on other entrancechannel properties like entrance-channel mass asymmetry [16], neutron richness, and deformation of the reaction partners [17-19]. However, the mechanism and properties of QF are still not well understood and we need more systematic studies to gain a better understanding.

Since closed-shell nuclei are used as target and/or projectile for SHE production, it is important to investigate their role in the reaction dynamics, and more specifically on QF. Work by Simenel et al. [20] showed the effect on QF of the total number of closed shells in the two colliding nuclei (defined there as "magicity"), the results suggesting that quasifission is reduced when more magic numbers are involved in the entrance channel, i.e., maximum QF reduction when both the target and projectile have closed-shell $N$ and $Z$. To isolate the influence of magic numbers, we need to eliminate effects due to other parameters that are known to influence the reaction dynamics, such as entrance-channel mass asymmetry, charge product $Z_{p} Z_{t}$, fissility and deformation of the heavy reaction partner.

In this study, we also investigate in detail the dependence of $\mathrm{QF}$ on the $N / Z$ values of the projectile and target nuclei. For this purpose, a set of reactions $\left({ }^{50,52,54} \mathrm{Cr}+{ }^{204,206,208} \mathrm{~Pb}\right)$ were carefully selected. Since the nuclei ${ }^{204,206,208} \mathrm{~Pb}$ are closed shell or nearly closed shell, they are essentially spherical in nature [21]. This eliminates the possibility of influence of target deformation. QF characteristics were studied using the mass-angle distribution (MAD) method. Mass-angle distributions have long been used to study quasifission [13,14,22], in particular the differences in timescales of CNF [23] and QF [13,14,24,25]. CNF occurs on a longer timescale [23] compared to deep inelastic [26] and quasifission [27]. In CNF, the formation of a compact compound nucleus "erases" any memory of the masses and directions of the colliding nuclei; thus, no correlation of mean fragment mass with angle remains.

In the case of $\mathrm{QF}$, the timescale is short and a complete rotation may not take place $[14,24]$. This gives rise to a correlation between mass and angle of the fission fragments. In general, the faster the quasifission, the stronger is the correlation in the MAD and wider is the mass distribution.

In Sec. II, we discuss experimental detail followed by the analysis and results in Sec. III. The interpretation of the obtained results is given in Sec. IV, and the conclusions are given in Sec. V.

\section{EXPERIMENT}

The experiments were carried out at the Australian National University, using the 14 UD tandem electrostatic accelerator followed by superconducting linear post-accelerator. Pulsed
TABLE I. Target thickness.

\begin{tabular}{lccc}
\hline \hline Target & Backing & $\begin{array}{r}\text { Backing thickness } \\
\left(\mu \mathrm{g} / \mathrm{cm}^{2}\right)\end{array}$ & $\begin{array}{r}\text { Target thickness } \\
\left(\mu \mathrm{g} / \mathrm{cm}^{2}\right)\end{array}$ \\
\hline${ }^{204} \mathrm{~Pb}$ & ${ }^{n a t} \mathrm{C}$ & 20 & 400 \\
${ }^{206} \mathrm{~Pb}$ & ${ }^{n a t} \mathrm{C}$ & 20 & 160 \\
${ }^{208} \mathrm{~Pb}$ & ${ }^{\text {nat }} \mathrm{C}$ & 20 & 150 \\
\hline \hline
\end{tabular}

beams of ${ }^{50,52,54} \mathrm{Cr}$ in the energy range of 257 to $292.7 \mathrm{MeV}$ were used for these measurements. Isotopically enriched targets of lead sulfide were mounted on a target ladder whose normal was rotated by an angle of $60^{\circ}$ with respect to the beam direction to avoid shadowing the fission detectors [15]. Target thicknesses are given in Table I.

Five reactions were measured in this work, namely ${ }^{50} \mathrm{Cr}+$ ${ }^{208} \mathrm{~Pb},{ }^{52} \mathrm{Cr}+{ }^{206,208} \mathrm{~Pb},{ }^{54} \mathrm{Cr}+{ }^{204,208} \mathrm{~Pb}$, which form different isotopes of $\operatorname{Sg}(Z=106)$. The bombarding energies in this work ranged between $E / V_{B}=0.97$ and $E / V_{B}=1.09$, where $E$ is center-of-mass energy and $V_{B}$ is the capture barrier of the corresponding reaction calculated based on systematics [28]. The experiment was performed using the CUBE spectrometer [29]. Two fission fragments were detected in coincidence using two large-area position-sensitive multiwire proportional counters (MWPC) of area $28 \times 36 \mathrm{~cm}^{2}$. The angular coverage of the detectors were $5^{\circ}$ to $80^{\circ}$ and $50^{\circ}$ to $125^{\circ}$. Two silicon surface barrier detectors were mounted at $22.5^{\circ}$ on the either side of beam direction for beam monitoring. The details of the experimental setup can be found in earlier papers [15,29].

\section{ANALYSIS AND RESULTS}

\section{A. Extraction of MADs and mass-ratio spectra}

The position and time information, obtained from the MWPC detectors, were initially converted into velocities in the laboratory frame. Assuming binary events, the velocity of the center-of-mass frame $\left(v_{\|}\right)$was determined [30] for each event. Gating on the strong peak at the $v_{\|}$expected for each $\mathrm{Cr}+\mathrm{Pb}$ measurement, reactions with light impurities could be completely rejected. The fragment velocities in the center-of-mass frame were then determined. The mass ratio is defined as $M_{R}=m_{1} /\left(m_{1}+m_{2}\right)$, where $m_{1}$ and $m_{2}$ are masses of the fission fragments at scission. The value of $M_{R}$ was obtained from momentum conservation and is given by

$$
M_{R}=v_{2} /\left(v_{1}+v_{2}\right),
$$

where $v_{1}, v_{2}$ are center-of-mass velocities of the fission fragments. The center-of-mass angle $\left(\theta_{\text {c.m. }}\right)$ was also determined for each event and mass-angle distributions (MAD) were then extracted for all the reactions as shown in Figs. 1 and 2. The elastic and inelastic events can be seen as intense groups near $M_{R}=0.2$ and 0.8 .

Mass-ratio distributions were extracted from these MAD spectra by projecting on the mass-ratio axis. For the heaviest fission fragments, traveling to the most backward angles, detection efficiency was reduced due to low signal height. This is visible in the MAD plots (Figs. 1 and 2) as a reduction in counts for $\theta_{\text {c.m. }} \geqslant 135^{\circ}$ and $M_{R} \backsim 0.7$. In order to avoid this low-efficiency region, and biasing due to detector geometry, 

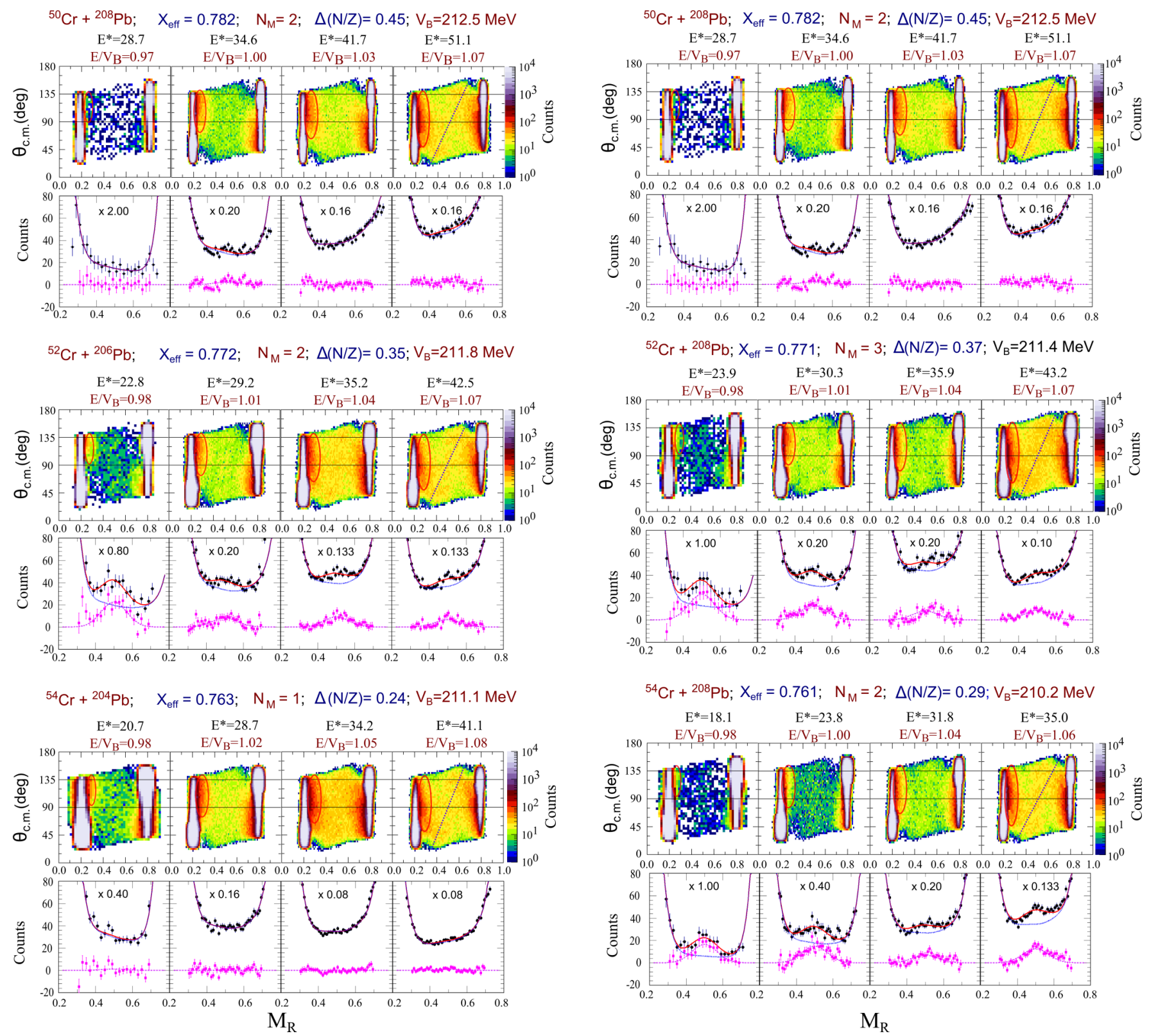

FIG. 1. MAD and mass ratio distribution for reactions leading to $\mathrm{CN}^{258} \mathrm{Sg}$. For a given reaction (arranged in a row), the energy increases to the right. Excitation energy $\left(E^{*}\right), E / V_{B}$, fissility ( $\left.\chi_{\text {eff }}\right)$, entrance channel magicity $\left(N_{M}\right), N / Z$ asymmetry $[\Delta(N / Z)]$, and capture barrier $\left(V_{B}\right)$ are given on the top of each MAD (see text). Fast quasifission events are marked by the red solid ellipse. Dashed lines on MADs at the highest energies guide the eye for mass-angle correlation of the slower QF component. The mass-ratio projections for each MAD are given below. The black circles are experimental mass ratio and the red lines show the best fitted curves. The background functions are shown by blue lines. Magenta circles and lines show extracted and fitted symmetric components, respectively.

an angular cut of $90^{\circ} \leqslant \theta_{\text {c.m. }} \leqslant 135^{\circ}$ was applied to obtain the mass distributions. These cuts are shown by the black lines in the MAD spectra. In general, because of their high energy loss, $\mathrm{Cr}+\mathrm{Pb}$ reaction products that entered the detectors, from DIC to symmetric fission, were detected with very high efficiency.

FIG. 2. Same as Fig. 1 for ${ }^{50,52,54} \mathrm{Cr}+{ }^{208} \mathrm{~Pb}$.

\section{B. Features of the MAD spectra}

We first discuss the nature of the obtained mass-angle distributions. MADs for ${ }^{50} \mathrm{Cr}+{ }^{208} \mathrm{~Pb},{ }^{52} \mathrm{Cr}+{ }^{206} \mathrm{~Pb}$, and ${ }^{54} \mathrm{Cr}+$ ${ }^{204} \mathrm{~Pb}$ are shown in Fig. 1 for four beam energies. These spanned the calculated [28] capture barrier energies $V_{B}$ as indicated. All these reactions form the same compound nucleus ${ }^{258} \mathrm{Sg}$. Figure 2 shows MADs for reactions of ${ }^{50,52,54} \mathrm{Cr}$ with the same target nucleus ${ }^{208} \mathrm{~Pb}$, in the energy range $E / V_{B}=0.97$ to 1.07 . Here, the ${ }^{50} \mathrm{Cr}+{ }^{208} \mathrm{~Pb}$ plots are the same as in Fig. 1. They are reproduced in Fig. 2 for easy comparison.

As a function of $E / V_{B}$, the main features of the MAD show a consistent systematic behavior. The quasielastic scattering (also including deep inelastic scattering with little mass change) at $M_{R} \simeq 0.2$ and 0.8 gives the largest yield. At the highest $E / V_{B}$, this decreases significantly at angles 


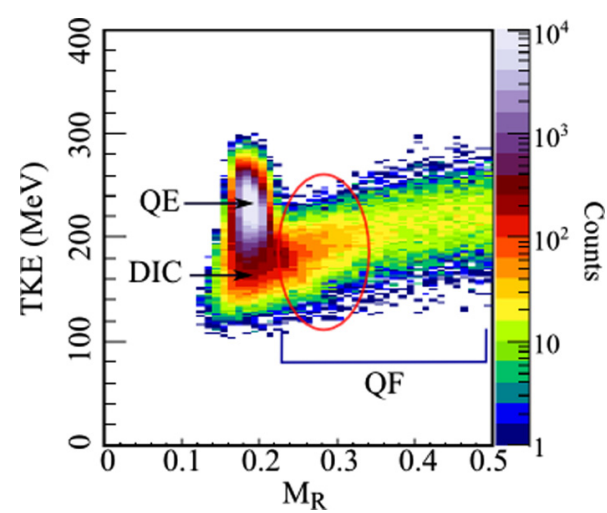

FIG. 3. Total kinetic energy as a function of $M_{R}$ for the reaction ${ }^{50} \mathrm{Cr}+{ }^{208} \mathrm{~Pb}$ at $E / V_{B}=1.07$. The quasielastic (QE), deep inelastic (DIC), and quasifission (QF) are indicated. Fast quasifission is highlighted by the red ellipse as in Figs. 1 and 2.

backward of $120^{\circ}$ and for $M_{R}$ value 0.2 (projectile-like nuclei) as increasing cross sections for energy-dissipative processes including capture deplete this component. Apart from the fission component peaked at mass symmetry $\left(M_{R}=0.5\right)$ discussed later for some reaction, there are two distinct features in all MADs: (i) a component with a small rotation angle and mass change $\left(M_{R} \leqslant 0.3\right.$ and $\geqslant 0.7$ for the complementary fragment) that is consistent with short sticking time and (ii) events with larger rotation angle and mass change (spanning $M_{R}=0.3$ to 0.7 ) associated with longer sticking time, yet still showing a strong mass-angle correlation associated with quasifission. At the lowest energies, the fast component is seen at the most backward angles covered by the detectors (120-135 ${ }^{\circ}$ for the projectile-like fragment), as indicated by the ellipses drawn to guide the eye. As the energy increases, the fast component spreads toward forward angles, as far as $45^{\circ}$ in the case of the highest energies. The small mass change and rotation angle indicates this component results from a short sticking time.

The nature of this component can be clarified by investigating the correlation of mass ratio with total kinetic energy (TKE). An example for ${ }^{50} \mathrm{Cr}+{ }^{208} \mathrm{~Pb}$ at $E / V_{B}=1.07$ is shown in Fig. 3, where the ellipse highlights the fast component. The TKE distribution is continuous with those of more symmetric quasifission, and thus appears to correspond to fully damped events. The mass flow toward symmetry and the essentially full damping of kinetic energy justify in our view the label fast quasifission. However, these events merge with fully damped binary events with little mass change (deep inelastic), which in turn merge into the partially damped and then quasielastic events, indicating that these physical process evolve smoothly from one to the other [15].

In addition to the fast $\mathrm{QF}$ yield, a substantial contribution from symmetric-peaked fission is seen in the region $0.3<$ $M_{R}<0.7$ for a few reactions (e.g. ${ }^{52} \mathrm{Cr}+{ }^{208} \mathrm{~Pb}$ in Fig. 2) at low beam energy. The lack of mass-angle correlation (within experimental uncertainty) for these events indicates that these are from slower quasifission and/or compound nuclear fission, contrasting with the faster quassifission components that shows

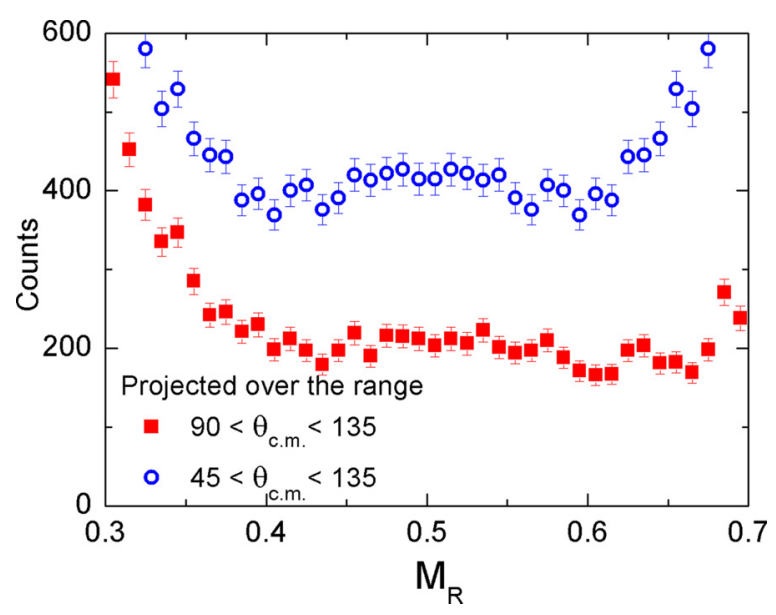

FIG. 4. Comparison of $M_{R}$ distribution when projected over the two different $\theta_{\text {c.m. }}$ range for the reaction ${ }^{52} \mathrm{Cr}+{ }^{206} \mathrm{~Pb}$.

a strong mass-angle correlation. This symmetric fission component will be discussed in detail in Sec. IV.

\section{Mass ratio distribution spectra}

The $M_{R}$ distributions, shown in Figs. 1 and 2, are obtained by projecting MADs on the $M_{R}$ axis. Figure 4 shows two mass-ratio distributions, which are obtained by projecting MAD over the angular range of $90^{\circ} \leqslant \theta_{\text {c.m. }} \leqslant 135^{\circ}$ and $45^{\circ} \leqslant$ $\theta_{\text {c.m. }} \leqslant 135^{\circ}$. For the angular cut $45^{\circ} \leqslant \theta_{\text {c.m. }} \leqslant 135^{\circ}$, the shape of the background becomes symmetric due to mirroring [29]. In principle, this could lead to a spurious symmetric component by mirroring a peak located away from $M_{R}=0.5$. Because of this, an asymmetric cut of $90^{\circ} \leqslant \theta_{\text {c.m. }} \leqslant 135^{\circ}$ was applied for the further study in order to avoid the generation of any spurious symmetric feature. The projected $M_{R}$ spectra (on a slightly extended $M_{R}$ scale) are shown in Figs. 1 and 2 below the corresponding MADs. The quasifission mass-ratio spectra show a "U"-shaped distribution. This is consistent with the systematic study by du Rietz et al. [15], where MADs were divided into three categories, namely MAD1, MAD2, and MAD3. The shortest timescale reactions (MAD1) are expected for entrance channel charge product $Z_{p} Z_{t}>1800$ and are empirically characterized by a "U"-shaped mass yield. For $\mathrm{Cr}+\mathrm{Pb}$ reactions, $Z_{p} Z_{t}$ is 1968 , which therefore fit into the category of MAD1 [15]. For ${ }^{50} \mathrm{Cr}+{ }^{208} \mathrm{~Pb}$ and ${ }^{54} \mathrm{Cr}+{ }^{204} \mathrm{~Pb}$ (Fig. 1), the spectra are similar at the same $E / V_{B}$. The slope of yield versus $M_{R}$ around $M_{R}=0.5$ is correlated with the mean angle of emission of the mass asymmetric fast quasifission fragments. As the angle changes with $E / V_{B}$, the slope changes as well for the given angular range. For these reactions, there is no evidence of a symmetric peak corresponding to mass-symmetric fission. In contrast with the above reactions, ${ }^{52} \mathrm{Cr}+{ }^{208} \mathrm{~Pb}$ shows a peak at mass symmetry at the lowest $E / V_{B}$. The mass-ratio spectra for higher $E / V_{B}$ also suggest a peaked mass-symmetric component on a background of events which show similar trends with energy to the ${ }^{50} \mathrm{Cr}+{ }^{208} \mathrm{~Pb}$ and ${ }^{54} \mathrm{Cr}+{ }^{204} \mathrm{~Pb}$ reactions.

In order to quantify the symmetric peaked yield, we need to determine the yields of the "U"-shaped fast quasifission 
component which overlap with the symmetric-peaked fission component. Hence, we fitted the experimental mass distributions with a fast quasifission background function combined with a Gaussian distribution having centroid at $M_{R}=0.5$. We assume that the QF has the same functional form at the same $E / V_{B}$ for all the reactions. The form of the background function was chosen such that it can fit mass distributions of the reaction ${ }^{54} \mathrm{Cr}+{ }^{204} \mathrm{~Pb}$ at all energies. This reaction does not show any peaklike structure even at the lowest energy, indicating that symmetric fission in this reaction has a very small contribution. For the rest of the reactions, the same background shape was used to estimate the symmetric fission contribution with respect to ${ }^{54} \mathrm{Cr}+{ }^{204} \mathrm{~Pb}$. It should be noted that the background function is not symmetric around $M_{R}=$ 0.5 because the experimental mass distributions were obtained by projecting the data for the angular range $90^{\circ} \leqslant \theta_{\text {c.m. }} \leqslant$ $135^{\circ}$. So, we need a background function that would also be asymmetric in nature and would not generate a spurious symmetric peak after background subtraction. The detail of the fitting of the background function is given in the appendix. The standard deviation of the Gaussian peak was fixed at 0.07 , which is consistent with other neighboring systems where a symmetric fission contribution is significant [20,31]. The fitted functions are shown in Figs. 1 and 2: The red solid curves represent the total fit and the blue dashed curves represent the fast quasifission background. When subtracted, this reveals any residual symmetric fission contribution, shown by magenta circles. The symmetric-peaked fitted Gaussian functions are shown by the magenta dash-dotted lines.

\section{INTERPRETATION}

\section{A. Reactions forming ${ }^{258} \mathrm{Sg}$}

To investigate the role of different entrance channel parameters, first we will discuss the three reactions ${ }^{50} \mathrm{Cr}+{ }^{208} \mathrm{~Pb}$, ${ }^{52} \mathrm{Cr}+{ }^{206} \mathrm{~Pb}$, and ${ }^{54} \mathrm{Cr}+{ }^{204} \mathrm{~Pb}$, which form the same compound nucleus ${ }^{258} \mathrm{Sg}$. MADs for these three reactions (shown in Fig. 1) are arranged so that reaction with similar $E / V_{B}$ are in same column. The values of excitation energy $E^{*}, E / V_{B}$, entrance channel magicity, effective fissility [15], and $N / Z$ target-projectile asymmetry which is defined as $\Delta(N / Z)=$ $(N / Z)_{t}-(N / Z)_{p}$ are given on the top of each row. All the reactions show a similar type of mass-angle correlation with a strong presence of mass asymmetric quasifission. It is clear from Fig. 1 that only the mass distributions for ${ }^{52} \mathrm{Cr}+{ }^{206} \mathrm{~Pb}$ show a peak at $M_{R}=0.5$ while the other two reactions do not show any peak even at the lowest energies. The mass symmetric fission yields are defined as the area under the peak in the range of $M_{R}=0.5 \pm 0.07$. This range corresponds to the standard deviation of the symmetric-peaked Gaussian used in the fitting. The symmetric fission $(P)$ to total fission (including QF) $(T)$ ratios $(P / T)$ were then extracted for all the mass distributions to get a measure of the contribution from slow fission to the total mass-symmetric fission-like events. Figure 5 shows the $P / T$ values of all the reactions as a function of $E / V_{B} \cdot{ }^{50} \mathrm{Cr}+{ }^{208} \mathrm{~Pb}$ and ${ }^{54} \mathrm{Cr}+{ }^{204} \mathrm{~Pb}$ have very small $P / T$ values which do not show any energy dependence, whereas ${ }^{52} \mathrm{Cr}+{ }^{206} \mathrm{~Pb}$ yielded higher $P / T$ values over the entire energy range compared

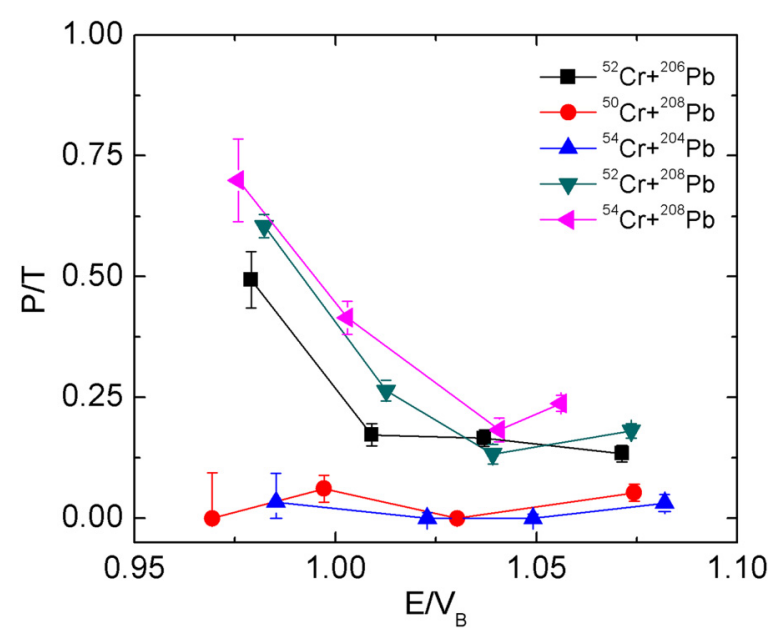

FIG. 5. Symmetric-peaked fission $(P)$ to total fission $(T)$ ratio (in the range of $0.43 \leqslant M_{R} \leqslant 0.57$ ) for reactions of $\mathrm{Cr}$ with $\mathrm{Pb}$. Lines guide the eye.

to other two reactions indicating a higher contribution from symmetric fission.

To summarize, the same $\mathrm{CN}$, formed at similar excitation energy or $E / V_{B}$ with the comparable entrance channel mass asymmetry, having identical $Z_{p} Z_{t}$, shows different fractions of symmetric-peaked fission. This indicates the influence of entrance-channel parameters other than charge product, deformation, and mass asymmetry. The effective fissility values $\left(\chi_{\text {eff }}\right)$ for ${ }^{50} \mathrm{Cr}+{ }^{208} \mathrm{~Pb},{ }^{52} \mathrm{Cr}+{ }^{206} \mathrm{~Pb}$, and ${ }^{54} \mathrm{Cr}+{ }^{204} \mathrm{~Pb}$ are $0.782,0.772$, and 0.763 , suggesting that $\chi_{\text {eff }}$ also cannot explain the higher $P / T$ in ${ }^{52} \mathrm{Cr}+{ }^{206} \mathrm{~Pb}$. Out of these three reactions, ${ }^{50} \mathrm{Cr}+{ }^{208} \mathrm{~Pb}$ and ${ }^{52} \mathrm{Cr}+{ }^{206} \mathrm{~Pb}$ have two magic numbers in the entrance channel and the third one has only one magic number, in the target. By considering the entrance channel magicity $N_{M}$ (the total number of magic numbers in target and projectile), the higher yield of symmetric fission in ${ }^{52} \mathrm{Cr}+{ }^{206} \mathrm{~Pb}$, compared to that of ${ }^{54} \mathrm{Cr}+{ }^{204} \mathrm{~Pb}$, can be justified. However, the reaction ${ }^{50} \mathrm{Cr}+{ }^{208} \mathrm{~Pb}$ shows no symmetric fission peak, though it has the doubly magic target ${ }^{208} \mathrm{~Pb}$. However, if we compare the $\Delta(N / Z)$ values for the three reactions, ${ }^{50} \mathrm{Cr}+{ }^{208} \mathrm{~Pb}$ has the highest $\Delta(N / Z)$ among these three reactions. Therefore, the symmetric fission yield in these reactions can not be explained solely by considering one of the above-mentioned parameters. It seems the magicity along with the difference in $N / Z$ between projectile and target nuclei decides the quasifission dynamics as proposed in Ref. [20].

\section{B. Reactions with ${ }^{208} \mathrm{~Pb}$}

To investigate the reaction dynamics further, we consider the three reactions on the same target ${ }^{208} \mathrm{~Pb}$ with different projectiles ${ }^{50,52,54} \mathrm{Cr}$ (Fig. 2), forming different isotopes of $\mathrm{Sg}$. At lower energies, the ${ }^{52} \mathrm{Cr}$ - and ${ }^{54} \mathrm{Cr}$-induced reactions show a peak near $M_{R}=0.5$ which becomes less visible at higher energies. Figure 5 shows that the $P / T$ values at the lowest energies are very high $(>0.50)$ for ${ }^{54} \mathrm{Cr}+{ }^{208} \mathrm{~Pb}$ and ${ }^{52} \mathrm{Cr}+{ }^{208} \mathrm{~Pb}$ and fall rapidly with increase in beam energy, whereas ${ }^{50} \mathrm{Cr}+{ }^{208} \mathrm{~Pb}$ has the lowest $P / T$ values over the 


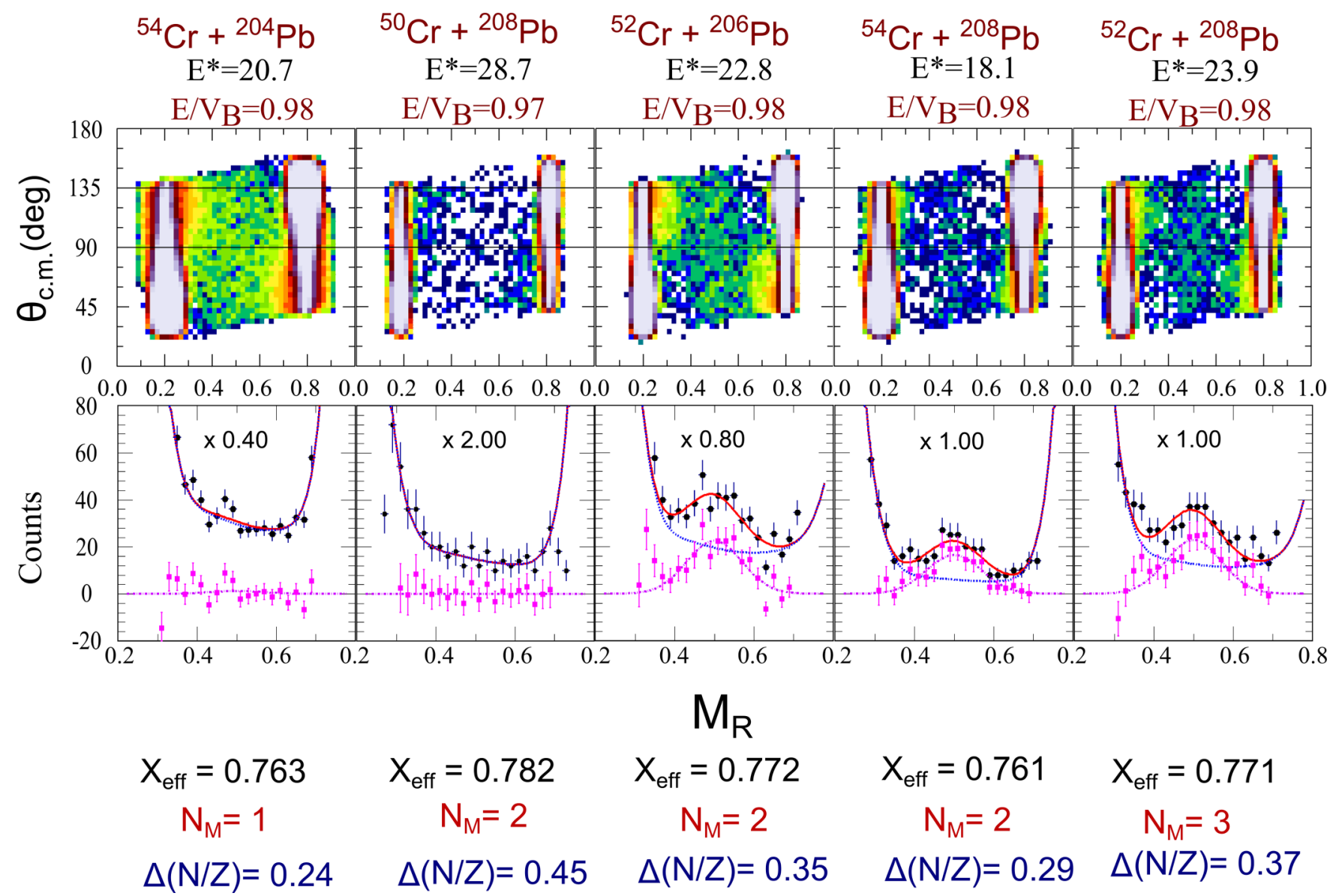

FIG. 6. Same as Fig. 1 for all $\mathrm{Cr}+\mathrm{Pb}$ reactions at similar below-barrier energies $\left(E / V_{B} \sim 0.98\right)$. The reactions are ordered from left to right, first by the numbers of magic numbers $N_{M}$, then by the $N / Z$ asymmetry, i.e., decreasing $\Delta(N / Z)$.

entire energy range and does not change with $E / V_{B}$. The reaction ${ }^{52} \mathrm{Cr}+{ }^{208} \mathrm{~Pb}$ has $N_{M}=3$ and very high $P / T$ values, in agreement with the idea that entrance-channel magic numbers influence the reaction dynamics. On the other hand, the two reactions ${ }^{50,54} \mathrm{Cr}+{ }^{208} \mathrm{~Pb}$ have the same $N_{M}$ value but ${ }^{50} \mathrm{Cr}+{ }^{208} \mathrm{~Pb}$ has a negligible amount of symmetric fission. $N / Z$ asymmetry $\Delta(N / Z)$ is less in ${ }^{54} \mathrm{Cr}+{ }^{208} \mathrm{~Pb}$ compared to ${ }^{50} \mathrm{Cr}+{ }^{208} \mathrm{~Pb}$. Comparison of these reactions is also consistent with the picture that the $N / Z$ asymmetry and number of closed-shell configurations are both deciding factors for the QF dynamics.

\section{Dependence on entrance channel characteristics}

To present most clearly the role played by different entrance-channel parameters, we arrange all the five reactions at the lowest and highest measured energies in Figs. 6 and 7 , respectively. The MADs are arranged first according to increasing $N_{M}$ values; for cases where $N_{M}$ is the same they are ordered by decreasing $\Delta(N / Z)$. It is clear that the symmetric peak is more prominent for reactions that have higher $N_{M}$ and smaller $\Delta(N / Z)$. This trend is present at both below- and above-barrier energies, but at the sub-barrier energies (Fig. 6) the contributions of the symmetric peaks are more visible. Quantitatively, this is seen in the $P / T$ values in Fig. 5.
The $P / T$ ratios for all the five reactions, at the lowest energies, are shown as a function of $N_{M}$ in Fig. 8. There are three reactions with $N_{M}=2$ showing wide variation in $P / T$ values but there is a correlation as $P / T$ is higher for reactions with smaller $\Delta(N / Z)$.

\section{Magic numbers}

By selecting reactions with similar $\Delta(N / Z)$, the effect of $N_{M}$ can be isolated. Figure 9 shows $P / T$ (the symmetricpeaked to total yield in the range of $0.43 \leqslant M_{R} \leqslant 0.57$ ) as a function of $N_{M}$. For energies below $V_{B}$ [Fig. 9(a)], the two systems with the smallest $\Delta(N / Z) \sim 0.25$ show a rise from $\lesssim 0.10$ to $0.74 \pm 0.10$ as $N_{M}$ increases from 1 to 2 . For $\Delta(N / Z) \sim 0.35$, there is a smaller rise from $N_{M}=2$ to 3 , with slightly smaller values of $P / T$. At the highest beam energies, Fig. 9(b) shows qualitatively the same effect, but the values of $P / T$ are approximately three times smaller.

The increase in symmetric-peaked fission with $N_{M}$ is in agreement with the results and conclusion in Ref. [20], where higher entrance-channel magicity was suggested to increase the symmetric fission contribution under the condition of good $N / Z$ matching. The effect of $N_{M}$ can be explained in terms of large gaps in the energy levels for closed-shell nuclei. The higher single-particle level spacing in closed-shell nuclei may 


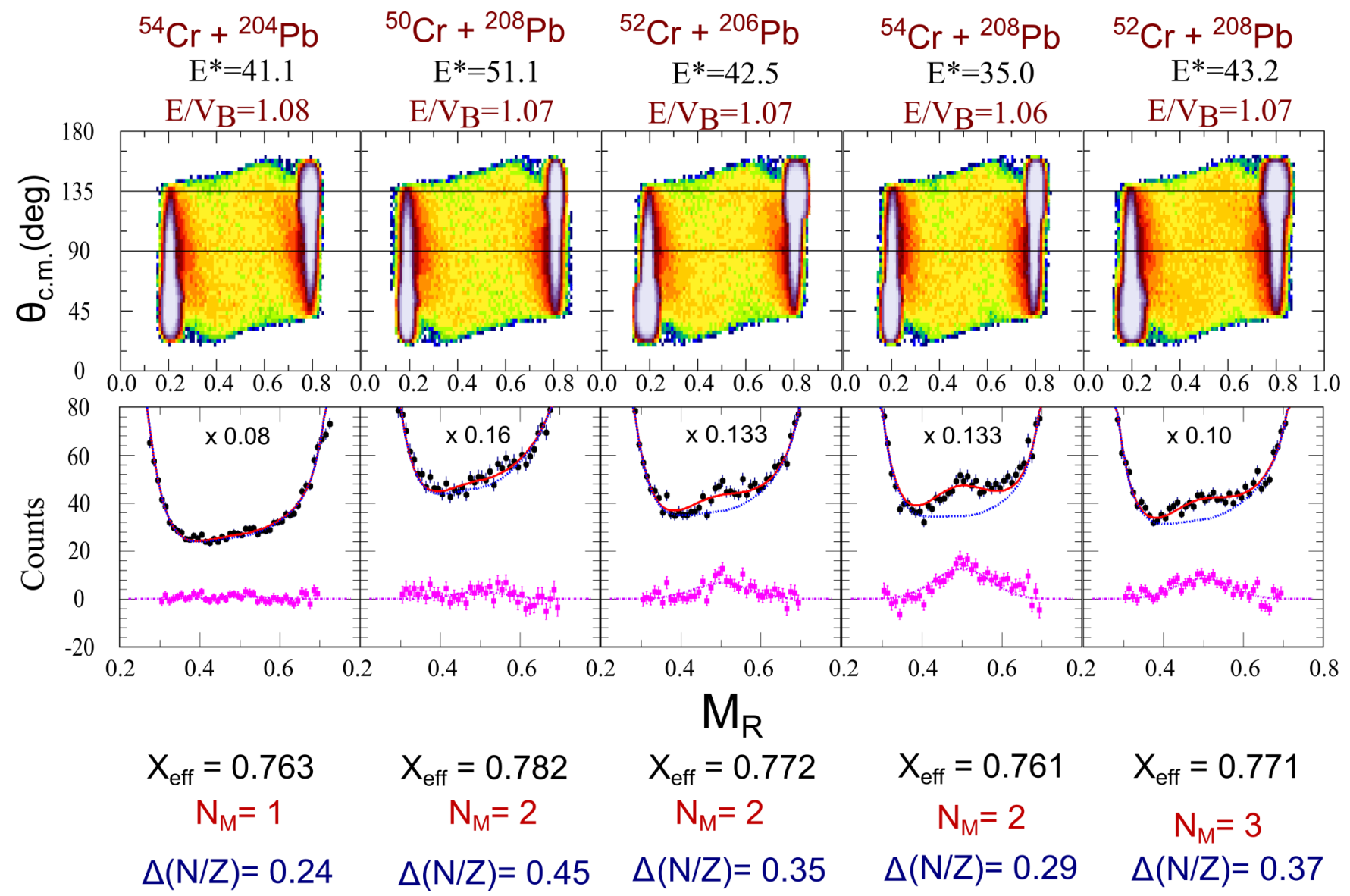

FIG. 7. Same as Fig. 1 for all $\mathrm{Cr}+\mathrm{Pb}$ reactions at similar above-barrier energies $\left(E / V_{B} \sim 1.07\right)$. The reactions are ordered in a way similar to those in Fig. 6.

reduce the dissipation of kinetic energy [32], resulting in a higher fusion probability.

With increasing beam energy, two effects might attenuate the influence of $N_{M}$. Higher average angular momentum would be expected to increase the probability of the shorter sticking time events (quasifission) at the expense of the

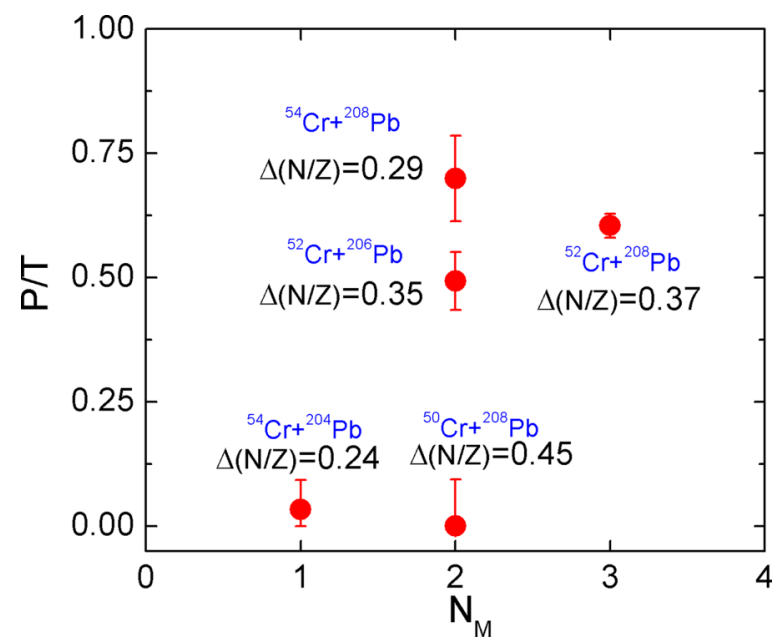

FIG. 8. Symmetric fission fraction at below-barrier energies as a function of magicity for all five reactions. long-lived symmetric-peaked fission (slow quasifission and/or fusion-fission). Even for the low-angular-momentum events, the increased relative velocity at contact (due to increasing energy) may increase the strength of energy dissipation and

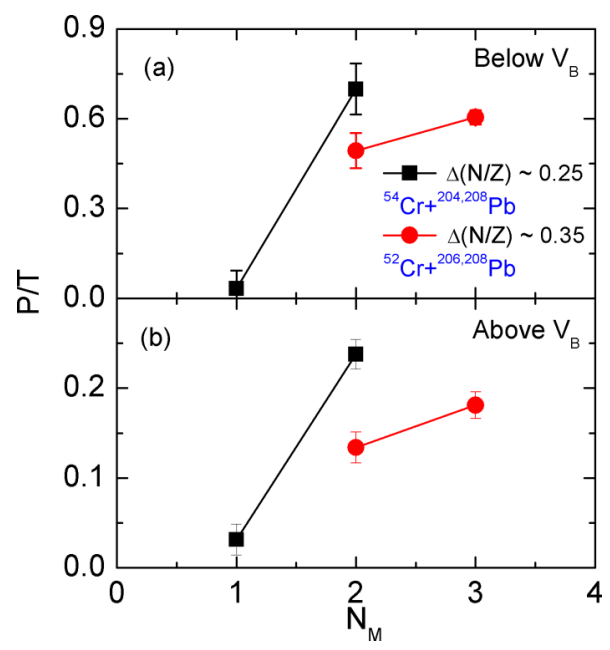

FIG. 9. Symmetric fission as a function of entrance channel magicity for the similar $N / Z$ asymmetry for reactions ${ }^{52,54} \mathrm{Cr}+$ ${ }^{208} \mathrm{~Pb},{ }^{52} \mathrm{Cr}+{ }^{206} \mathrm{~Pb}$, and ${ }^{54} \mathrm{Cr}+{ }^{204} \mathrm{~Pb}$ at similar energies, for $E / V_{B} \sim$ 0.98 (a) and 1.07 (b). 


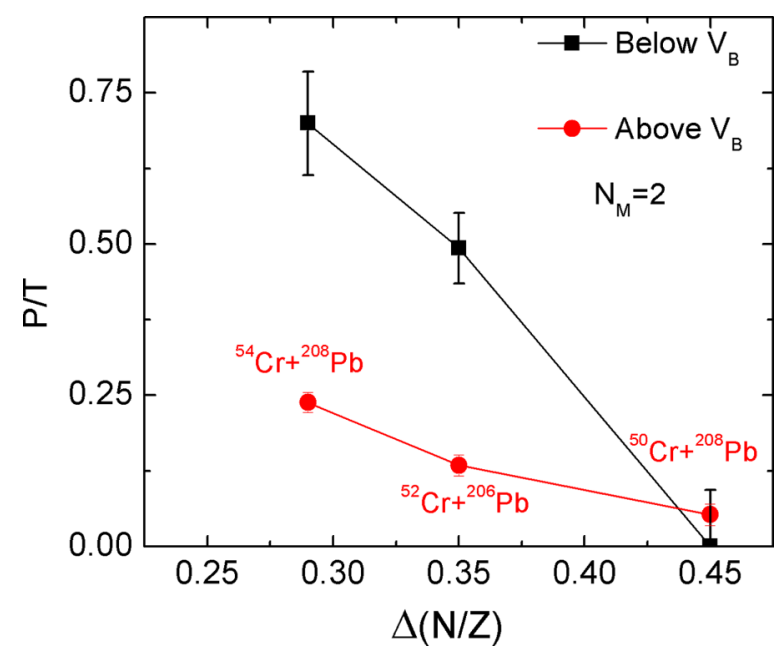

FIG. 10. Symmetric fission fraction vs $N / Z$ asymmetry for the same magicity $\left(N_{M}=2\right)$ for reactions ${ }^{50} \mathrm{Cr}+{ }^{208} \mathrm{~Pb},{ }^{52} \mathrm{Cr}+{ }^{206} \mathrm{~Pb}$, and ${ }^{54} \mathrm{Cr}+{ }^{208} \mathrm{~Pb}$.

thus reduce the probability of reaching more compact (longlived) configurations. Thus, the observed attenuation of $P / T$ with $E / V_{B}$ seems reasonable.

\section{2. $N / Z$ matching}

To focus on $N / Z$ asymmetry in the present set of reactions, we consider the three reactions ${ }^{50} \mathrm{Cr}+{ }^{208} \mathrm{~Pb},{ }^{52} \mathrm{Cr}+{ }^{206} \mathrm{~Pb}$, and ${ }^{54} \mathrm{Cr}+{ }^{208} \mathrm{~Pb}$, each having $N_{M}=2 . P / T$ for these three reactions is plotted as a function of $\Delta(N / Z)$ in Fig. 10. At below-barrier energies, the value of $P / T$ decreases with increasing $\Delta(N / Z)$ (shown by filled squares). The same trend is seen even for the highest energies (shown by filled circles) but overall the values of $P / T$ are smaller, as already discussed. A large value of $\Delta(N / Z)$ can completely eliminate the symmetric peaked fission.

The observed trends can be understood by considering that $\Delta(N / Z)$ is a good proxy for the relevant transfer reaction $Q$ values. As suggested in Ref. [20], transfer reactions at an early stage of the collision will act to attenuate the effects of the initial $N_{M}$ of the reaction partners by changing their identities. In Fig. 11 , we show $Q_{\mathrm{gg}}-Q_{\text {opt }}$ values $\left(Q_{\mathrm{gg}}\right.$ is the ground state $Q$ value and $Q_{\text {opt }}$ is the optimum $Q$ value) [33,34] as a function of $\Delta(N / Z)$. Where $Q_{\mathrm{gg}}-Q_{\mathrm{opt}}$ is positive, transfer to many excited states is kinematically favorable, and therefore a high transfer probability is expected. Where $Q_{\mathrm{gg}}-Q_{\mathrm{opt}}$ is negative, transfer is not favored, and the reactants are likely to retain their identity for longer.

For all five reactions mentioned here, stripping of $1 n$ or $2 n$ and pickup of $1 p$ or $2 p$ have negative $Q_{\mathrm{gg}}-Q_{\mathrm{opt}}$, indicating they will not contribute. The $1 n, 2 n$ pickup and $1 p, 2 p$ stripping $Q_{\mathrm{gg}}-Q_{\text {opt }}$ values tend to increase with $\Delta(N / Z)$. The ${ }^{54} \mathrm{Cr}+$ ${ }^{208} \mathrm{~Pb}$ reaction, with $N_{M}=2$, has all nucleon transfer $Q$-values negative, such that the $N / Z$ values of the projectile and target nuclei, though different, can be considered to be well matched. Consequently it shows a high proportion of symmetric-peaked fission (see Fig. 10). In contrast, for ${ }^{50} \mathrm{Cr}+{ }^{208} \mathrm{~Pb}\left(N_{M}=\right.$ 2 also), all $Q$ values plotted are positive, which correlates

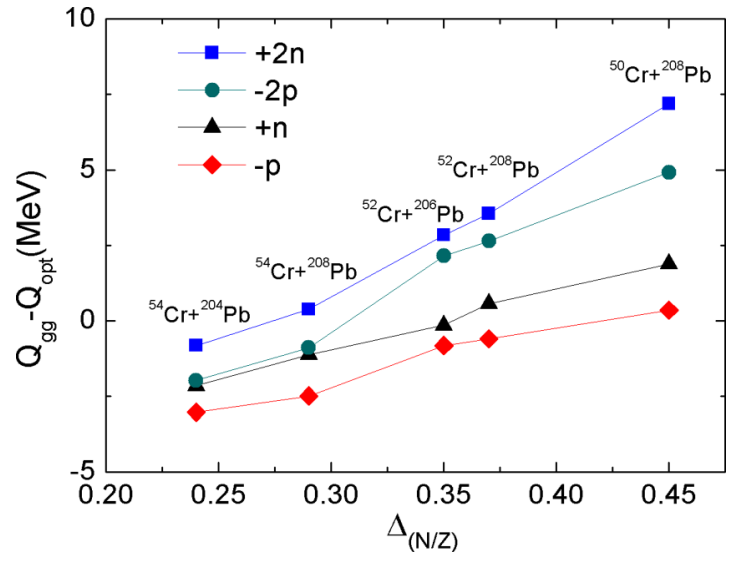

FIG. 11. $Q_{\mathrm{gg}}-Q_{\mathrm{opt}}$ of nucleon transfer channels with positive $Q$ values for the five $\mathrm{Cr}+\mathrm{Pb}$ reactions as a function of $\Delta(N / Z)$.

with the absence of any significant symmetric-peaked fission component.

\section{CONCLUSIONS}

In the present study, the effect of entrance-channel spherical closed shells and $N / Z$ asymmetry on quasifission dynamics has been studied for the reactions ${ }^{50,52,54} \mathrm{Cr}+{ }^{204,206,208} \mathrm{~Pb}$. By carefully selecting these reactions, we eliminate other entrance channel effects like charge product, entrance-channel mass asymmetry, deformation, fissility, etc. The mass distributions for all the five reactions were highly correlated with angle, indicating a strong presence of quasifission, with a fast timescale, which is in good agreement with the earlier reported systematics by du Rietz et al. [15]. At lower energies, a slower mass-symmetric component was also present for some of the reactions.

The deducted fractions of symmetric-peaked fission clearly show a sensitivity to both the number of entrance-channel spherical closed shells ("magicity") and the $N / Z$ mismatch between the target and projectile nuclei, the latter of which is strongly correlated with $Q_{\mathrm{gg}}-Q_{\mathrm{opt}}$ and therefore a propensity for transfer reactions. Large fractions of symmetric fission appear to require both higher magicity and small $\Delta(N / Z)$, consistent with earlier reports on $\mathrm{Ca}$ - and Ti-induced reactions [20]. Increased magicity allows for greater inter-penetration of collision partners, and a small $\Delta(N / Z)$ ensures the magicity is not completely destroyed by nucleon transfers early in the collision. If either the magicity is small or a large value of $\Delta\left(N / Z\right.$ ) drives nucleon transfer (as in ${ }^{50} \mathrm{Cr}+{ }^{208} \mathrm{~Pb}$ ), the fraction of symmetric fission is much reduced.

\section{ACKNOWLEDGMENTS}

The authors acknowledge the Australian Research Council for support through Discovery Grants No. DP140101337, No. DP160101254, No. FL110100098, No. DE140100784, No. FT120100760, and No. DP170102318. NCRIS is thanked for the operating support for the ANU Heavy Ion Accelerator Facility. 


\section{APPENDIX: EXTRACTING SYMMETRIC FISSION PART}

For extraction of the symmetric fission part, the mass ratio distributions which did not have any peak at $M_{R}=0.5$ were fitted with a background function having the form

$$
\begin{aligned}
f= & a_{0}\left\{1+a_{1}\left(M_{R}-0.5\right)+a_{3}\left(M_{R}-0.5\right)^{6}\right\}, \\
& \text { for } M_{R}<0.5 \\
= & a_{0}\left\{1+a_{1}\left(M_{R}-0.5\right)+a_{2}\left(M_{R}-0.5\right)^{2}\right. \\
& \left.+a_{4}\left(M_{R}-0.5\right)^{8}\right\}, \text { for } M_{R}>0.5,
\end{aligned}
$$

where $a_{0}, a_{1}, a_{2}, a_{3}$, and $a_{4}$ are free parameters. It is important to mention here that the exact choice of the background function need not be unique. It was chosen such that it can give reasonable fit at higher as well as lower $M_{R}$ values in the range $0.3 \leqslant M_{R} \leqslant 0.7$ of interest. Lower order terms $\left(M_{R}-0.5\right)$ and $\left(M_{R}-0.5\right)^{2}$ were included to fit the background function near $M_{R}=0.5$. The terms $\left(M_{R}-0.5\right)^{6}$ and $\left(M_{R}-0.5\right)^{8}$ were used to reproduce the elastic and deep-inelastic regions only. To obtain the symmetric fission events, the region from $M_{R}=$ $0.5 \pm 0.07$ was used, and over this region the contributions from these two terms are negligible. So, the choice of these two terms will not affect the symmetric fission extraction.

To obtain the symmetric fission contribution, the two parameters $a_{1}$ and $a_{2}$ are crucial and need to be chosen properly. The best-fit values of these two parameters showed definite trends with $E / V_{B}$ for most of mass distributions. The other two parameters $a_{3}$ and $a_{4}$ did not follow such trends as these two parameters depend on the resolution of the elastic peaks which can be affected by the target thickness. A simple energy dependence of the linear and quadratic coefficients [as shown in Figs. 12(a) and 12(b) respectively] was sought to define the shape of the fast quasifission component for all reactions.

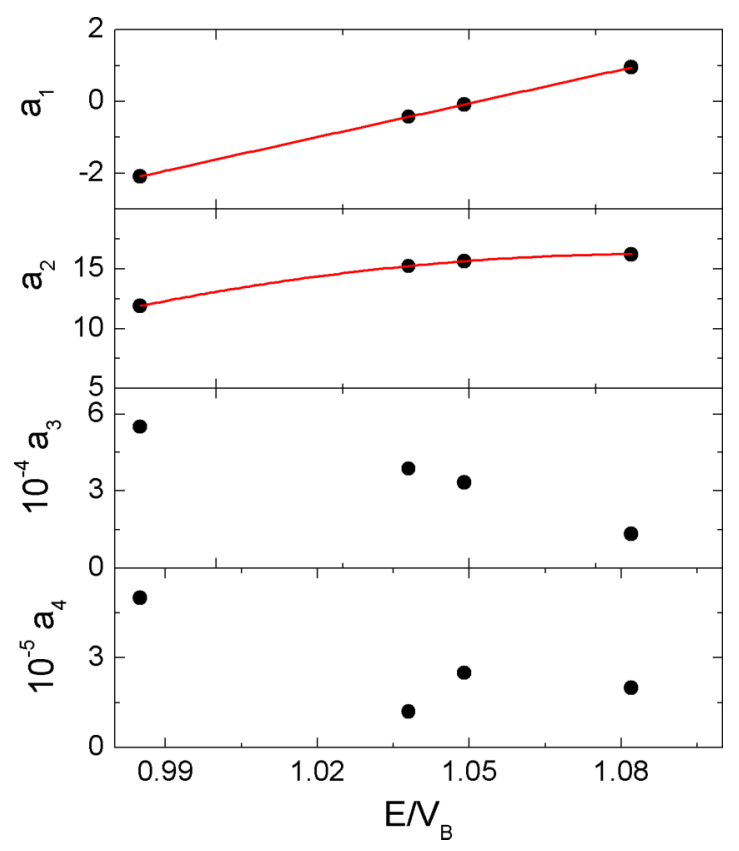

FIG. 12. Values of parameters $a_{1}, a_{2}, a_{3}, a_{4}$, used to fit the mass distributions for ${ }^{54} \mathrm{Cr}+{ }^{204} \mathrm{~Pb}$ reactions as a function of $E / V_{B}$. Red lines are the fitted values.

Linear and quadratic functions gave a good fit to the four ${ }^{54} \mathrm{Cr}+{ }^{204} \mathrm{~Pb}$ measurements. The average $\chi^{2}$ per degree of freedom of 1.37 for these four measurements confirmed the adequacy of this functional form. For reactions other than ${ }^{54} \mathrm{Cr}+{ }^{204} \mathrm{~Pb}$, the values of $a_{1}\left(E / V_{B}\right)$ and $a_{2}\left(E / V_{B}\right)$ were obtained by the fitted linear and parabolic functions and the remaining parameters $\left(a_{0}, a_{3}, a_{4}\right)$ were varied freely along with the contribution from the Gaussian peak.
[1] A. Sobiczewski, F. A. Gareev, and B. N. Kalinkin, Phys. Lett. 22, 500 (1966).

[2] S. Hofmann and G. Munzenberg, Rev. Mod. Phys. 72, 733 (2000).

[3] W. D. Myers and W. J. Swiatecki, Nucl. Phys. 81, 1 (1966).

[4] M. Bender, K. Rutz, P. G. Reinhard, J. A. Maruhn, and W. Greiner, Phys. Rev. C 60, 034304 (1999).

[5] Y. T. Oganessian, V. K. Utyonkov, Y. V. Lobanov, F. S. Abdullin, A. N. Polyakov, R. N. Sagaidak, I. V. Shirokovsky, Y. S. Tsyganov, A. A. Voinov, G. G. Gulbekian et al., Phys. Rev. C 74, 044602 (2006).

[6] Y. T. Oganessian, F. S. Abdullin, C. Alexander, J. Binder, R. A. Boll, S. N. Dmitriev, J. Ezold, K. Felker, J. M. Gostic, R. K. Grzywacz et al., Phys. Rev. Lett. 109, 162501 (2012).

[7] P. Armbruster, Rep. Prog. Phys. 62, 465 (1999).

[8] K. Morita, K. Morimoto, D. Kaji, T. Akiyama, S. Goto, H. Haba, E. Ideguchi, K. Katori, H. Koura, and H. Kudo, J. Phys. Soc. Jpn. 76, 043201 (2007).

[9] Y. Oganessian, J. Phys. G 34, R165 (2007).

[10] J. Khuyagbaatar, Phys. Rev. Lett. 112, 172501 (2014).

[11] Y. T. Oganessian and V. K. Utyonkov, Nucl. Phys. A 944, 62 (2015).
[12] Y. T. Oganessian and V. K. Utyonkov, Rep. Prog. Phys. 78, 036301 (2015).

[13] J. Tōke, R. Bock, G. Dai, A. Gobbi, S. Gralla, K. Hildenbrand, J. Kuzminski, W. Müller, A. Olmi, H. Stelzer et al., Nucl. Phys. A 440, 327 (1985).

[14] W. Q. Shen, J. Albinski, A. Gobbi, S. Gralla, K. D. Hildenbrand, N. J. Kuzminski, W. F. J. Muller, H. Stelzer, and J. Toke, Phys. Rev. C 36, 1 (1987).

[15] R. du Rietz, E. Williams, D. J. Hinde, M. Dasgupta, M. Evers, C. J. Lin, D. H. Luong, C. Simenel, and A. Wakhle, Phys. Rev. C 88, 054618 (2013).

[16] B. B. Back, P. B. Fernandez, B. G. Glagola, D. Henderson, S. Kaufman, J. G. Keller, S. J. Sanders, F. Videbaek, T. F. Wang, and B. D. Wilkins, Phys. Rev. C 53, 1734 (1996).

[17] D. J. Hinde, M. Dasgupta, J. R. Leigh, J. P. Lestone, J. C. Mein, C. R. Morton, J. O. Newton, and H. Timmers, Phys. Rev. Lett. 74, 1295 (1995).

[18] D. J. Hinde, R. G. Thomas, R. du Rietz, A. Diaz-Torres, M. Dasgupta, M. L. Brown, M. Evers, L. R. Gasques, R. Rafiei, and M. D. Rodriguez, Phys. Rev. Lett. 100, 202701 (2008).

[19] D. J. Hinde, R. du Rietz, M. Dasgupta, R. G. Thomas, and L. R. Gasques, Phys. Rev. Lett. 101, 092701 (2008). 
[20] C. Simenel, D. J. Hinde, R. du Rietz, M. Dasgupta, M. Evers, C. J. Lin, D. H. Luong, and A. Wakhle, Phys. Lett. B 710, 607 (2012).

[21] A. C. Berriman, D. J. Hinde, M. Dasgupta, C. R. Morton, R. D. Butt, and J. O. Newton, Nature (London) 413, 144 (2001).

[22] C. Ngo and H. Hofmann, Z. Phys. A 282, 83 (1977).

[23] D. J. Hinde, D. Hilscher, H. Rossner, B. Gebauer, M. Lehmann, and M. Wilpert, Phys. Rev. C 45, 1229 (1992).

[24] K. Lützenkirchen, J. V. Kratz, G. Wirth, W. Brüchle, L. Dörr, K. Sümmerer, R. Lucas, J. Poitou, C. Grégoire, and S. Bjørnholm, Z. Phys. A 320, 529 (1985).

[25] R. du Rietz, D. J. Hinde, M. Dasgupta, R. G. Thomas, L. R. Gasques, M. Evers, N. Lobanov, and A. Wakhle, Phys. Rev. Lett. 106, 052701 (2011).

[26] C. Gregoire, C. Ngo, and B. Remaud, Nucl. Phys. A 383, 392 (1982).
[27] L. Moretto and R. Schmitt, Rep. Prog. Phys. 44, 533 (1981).

[28] W. J. Swiatecki, K. Siwek-Wilczynska, and J. Wilczynski, Phys. Rev. C 71, 014602 (2005).

[29] R. G. Thomas, D. J. Hinde, D. Duniec, F. Zenke, M. Dasgupta, M. L. Brown, M. Evers, L. R. Gasques, M. D. Rodriguez, and A. Diaz-Torres, Phys. Rev. C 77, 034610 (2008).

[30] D. J. Hinde, M. Dasgupta, J. R. Leigh, J. C. Mein, C. R. Morton, J. O. Newton, and H. Timmers, Phys. Rev. C 53, 1290 (1996).

[31] G. N. Knyazheva, E. M. Kozulin, R. N. Sagaidak, A. Y. Chizhov, M. G. Itkis, N. A. Kondratiev, V. M. Voskressensky, A. M. Stefanini, B. R. Behera, L. Corradi et al., Phys. Rev. C 75, 064602 (2007).

[32] P. Armbruster, Annu. Rev. Nucl. Part. Sci. 50, 411 (2000).

[33] P. J. A. Buttle and L. J. B. Goldfarb, Nucl. Phys. A 176, 299 (1971).

[34] J. Wilczynski and H. W. Wilschut, Phys. Rev. C 39, 2475 (1989). 